\title{
LA AUTOEVALUACIÓN NO ES EL CAMINO DIRECTO PARA LA ACREDITACIÓN UNIVERSITARIA
}

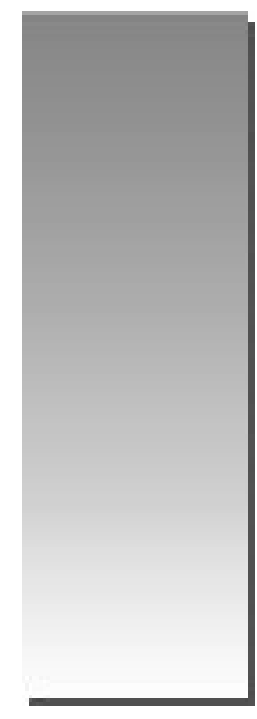

Herbert Valencia Pomareda*

E-mail: hvalenciap@unmsm.edu.pe

\begin{abstract}
RESUMEN
La acreditación universitaria busca, sobre todo, la calidad académica por lo que debemos conocer previamente las competencias laborales que los usuarios o empresas necesitan de nuestros egresados; por lo tanto, no es aconsejable lograr la acreditación por medio de una autoevaluación, ya que si nos autoevaluamos primero sin conocer las necesidades educativas de la sociedad puede ocurrir que los elementos evaluados no sean de importancia para lograr la calidad deseada.
\end{abstract}

Palabras clave: Acreditación, calidad académica, retroalimentación positiva, retroalimentación negativa.

\section{ABSTRACT}

The university accreditation looks for, mainly, the academic quality or labor competencies that our users or enterprises need from the leaving students. As a consequence, it is not advisable to get the accreditation by means of self-evaluation. To get the required quality, it is necessary to know the educational needs of the society.

Key words: Acreditación, academic quality, positive feedback, negative feedback.

\section{INTRODUCCIÓN}

La competencia también ha llegado a las universidades, por lo tanto, si se desea seguir en el mercado educativo, es necesario mejorar la calidad educativa. Si entendemos que la calidad es otorgar un producto o servicio de acuerdo a lo que realmente necesita el mercado, es importante e indispensable conocer con suma certeza qué es lo que requiere y necesita el mercado, como también el por qué del movimiento universitario hacia la calidad.
Según Depaz Toledo y Cuba Sancho en Lineamientos para una Política de calidad, autoevaluación y acreditación, se entiende por acreditación al reconocimiento y certificación por parte de un organismo competente, nacional o internacional, externo a la universidad, de que la institución o alguno de sus programas cumple con los estándares mínimos de calidad previamente establecidos. Su propósito es promover la calidad de la educación superior, hacerla merecedora de la confianza pública y adecuarla a las necesidades del país.

* Estudios de Maestría en Gestión Empresarial y Estudios en el Doctorado en Administración. Licenciado en Administración. Profesor Asociado de la Facultad de Ciencias Administrativas de la Universidad Nacional Mayor de San Marcos. 
De acuerdo a la Real Academia de la Lengua, acreditación significa:

"Hacer digno de crédito a algo, probar su certeza o realidad. / Afamar, dar crédito o reputación. / Dar seguridad de que alguien o algo es lo que representa o parece. / Dar testimonio en documento fehaciente de que alguien tiene facultades para desempeñar comisión o encargo diplomático, comercial, etc."

A través del proceso evolutivo histórico de las Escuelas de la Ciencia Administrativa, todas ellas con excepción de Escuela Sistémica y la Escuela Contingencial, toman en cuenta prioritariamente el contexto interno del "qué hacer" administrativo, sin tomar en cuenta el "por qué hacer", ubicados en el contexto externo en función de lo que la empresa quiera del futuro; para ello es importante conocer fehacientemente las facultades de desempeño laboral que requieren los usuarios.

\section{¿QUÉ ES LA ACREDITACIÓN UNIVERSITARIA?}

Viñas-Román define la acreditación como el reconocimiento social e institucional, de carácter temporal, mediante el cual se da fe pública de los méritos y el nivel de calidad de una institución de educación superior, de un programa, de alguna de sus funciones o de sus elementos constitutivos, que culmina con la certificación de la institución o programa evaluado, verificando que cumpla con los estándares de calidad pre establecidos.

Es necesario diferenciar acreditación de certificación. Se acreditan a las organizaciones o personas que emiten certificaciones o realizan servicios; en cambio, se certifican productos y servicios, sistemas de gestión o competencias laborales de una persona. El objetivo principal de la acreditación es contribuir a la mejora de la educación superior mediante la certificación a nivel nacional o internacional de programas e instituciones que cumplan requisitos de calidad establecidos.

\section{MOVIMIENTOS HACIA LA CALIDAD ACADÉMICA}

Como hemos podido observar la acreditación universitaria busca, fundamentalmente, la calidad académica que, como lo manifiestan Cabrejos Polo; Gutiérrez Huby y López Más en Hacia la excelencia Académica es "una filosofía de vida personal e institucional; es una manera de pensar y actuar, y guarda una relación directa con la cultura de la organización".

Por lo tanto, para lograr la calidad, la comunidad universitaria deberá estar comprometida con el cambio en el marco de una cultura de evaluación, transparencia, excelencia académica con políticas y un comportamiento organizacional de calidad. Si la calidad es una filosofía de vida, entonces ésta no se improvisa ni se impone; es un trabajo de cambio cultural que se conquista día a día y que constituye la ventaja competitiva más poderosa de cualquier organización.

Resumiendo a Depaz Toledo y Cuba Sancho, las razones del movimiento hacia la calidad son las siguientes:

- La humanidad ha ingresado a una visión holística, entendiendo como holismo a la doctrina que propugna la concepción de cada realidad como un todo distinto de la suma de las partes que la componen, o como la perspectiva que sitúa al sujeto o fenómeno en función al contexto o totalidad de la que hace parte, atendiendo al conjunto de sus aspectos.

- Tendencias de calidad en organizaciones industriales y de servicios.

- Mejor entendimiento de la influencia de la educación en el desarrollo de la nación.

- Por recomendación y promoción de la UNESCO.

- Globalización y movilización de los profesionales siendo su campo de acción el mundo, no solo sus países

\section{RETROALIMENTACIÓN POSITIVA VS. RETROALI- MENTACIÓN NEGATIVA}

Los avances cada vez más veloces en ciencia y tecnología, han logrado que se materialice el desarrollo acelerado de la sociedad de la información, trayendo consigo la necesidad de efectuar -en forma continua y permanente- procesos de transformación de la información en conocimiento relevante y utilizable para diferentes contextos donde sea útil aplicarlo para el desarrollo de las sociedades, sin perder el contexto externo.

Los cambios dados mediante la evolución de las ciencias administrativas (fundamentalmente en la Escuela Sistémica y la Escuela Contingencial), nos hacen entender la enorme y vital importancia que tienen los clientes para las organizaciones. 
Las escuelas iniciales de las Ciencias Administrativas daban demasiada importancia a los factores endógenos (internos) de las organizaciones. Se preocupaban en el ¿qué hacer?, ¿cómo hacer? y hasta en el ¿cuán bien hacer?, sin importarles mucho o poco los gustos y necesidades de los clientes. Este hacer, sin tomar en cuenta los factores exógenos (clientes externos) se conoce como "Retroalimentación Negativa" según Henry Sisk y Marco Sverdlik, en Administración y gerencia empresarial.

Según los mismos autores, cuando la empresa antes de pensar en el ¿qué hacer? ¿cómo hacer? y ¿cuán bien hacer? piensa primero en las necesidades de sus clientes, estará actuando con "Retroalimentación Positiva".

\section{LA RETROALIMENTACIÓN POSITIVA DETERMI- NA LA RETROALIMENTACIÓN NEGATIVA}

Comenzamos manifestando que la competencia también ha llegado a las universidades y para que éstas continúen en el mercado educativo deberán mejorar continuamente su calidad, por lo que es indispensable conocer con precisión las necesidades de educación de sus usuarios del futuro y adecuar sus recursos internos como calidad académica, eficacia de gestión y plan estratégico; de acuerdo a ello se podrán ofrecer los servicios que el usuario requiere. En otras palabras, la Retroalimentación Positiva deberá determinar el tipo de Retroalimentación Negativa que deberán tener las universidades.

Por lo tanto los nichos educativos y hasta segmentos de cualquier universidad no necesariamente serán los mismos. Por ejemplo, el nicho de mercado que tengan las facultades de Administración, Contabilidad y Economía serán las empresas, razón por lo que es necesario establecer una relación estrecha entre estas facultades, las universidades y la empresa, siendo necesario conocer qué tipo de profesionales en estas áreas requieren las empresas, y el perfil profesional de los egresados deberá efectuarse en coordinación con las empresas.

\section{LA AUTOEVALUACIÓN NO ES EL CAMINO DIREC- TO PARA LA ACREDITACIÓN}

Si entendemos que la acreditación universitaria busca fundamentalmente la calidad académica, entonces en lo que se deberá preocupar priori tariamente la universidad, antes de hacerse una autoevaluación, será en conocer las necesidades educativas y de acuerdo a ello recién internarse para determinar si sus factores endógenos (autoevaluación) son apropiados para satisfacer las necesidades de sus usuarios, pudiendo darse el caso que después de una autoevaluación exitosa, nos damos cuenta que los factores que hemos evaluado no son los necesarios y apropiados para satisfacer las necesidades de nuestros usuarios, estableciendo prioridad a la relación universidad-empresa.

\section{CONCLUSIONES}

- La autoevaluación no es el camino directo para la acreditación universitaria.

- La acreditación universitaria busca fundamentalmente la calidad académica.

- La calidad académica será determinada únicamente por los clientes.

- Las universidades, al ofrecer una variedad de ofertas educativas (diferentes facultades y programas académicos), tendrán también diferentes nichos de mercado.

- La acreditación universitaria tiene carácter temporal.

- Mejor entendimiento de la influencia de la educación en el desarrollo de las naciones.

\section{BIBLIOGRAFÍA}

CABREJOS, JORGE; GUTIÉRREZ, ANA MARÍA y LÓPEZ MAS, JULIO. (2005). Hacia la excelencia académica. UNMSM.

CHIAVENATO, ADALBERTO. (2000). Procesos administrativos. Bogotá, Editorial Mc. Graw Hill.

DEPAZ TOLEDO, ZENÓN y SANCHO CUBA, JUANA. (2003). Lineamientos para una Política de calidad, Autoevaluación y Acreditación. Oficina Central de calidad Académica y Acreditación- UNMSM. Octubre 2003.

SISK, HENRY y SVERDLIK, MARCO. (1999). Administración y gerencia de empresas. South-Western Publishing.

VIÑAS-ROMÁN, JAIME. (1998). Ideas en torno a los procesos de mejoramiento de la Educación. Costa Rica, Centro de Educación y Capacitación (CECAP). 